\title{
Two-timescale adiabatic expansion of a scalar field model
}

\author{
Yasushi Mino* \\ Mail code 130-33, California Institute of Technology, Pasadena, California 91125, USA \\ Richard H. Price ${ }^{\dagger}$ \\ Center for Gravitational Wave Astronomy, 80 Fort Brown, Brownsville, Texas. 78520, USA
}

(Received 31 December 2007; published 3 March 2008)

\begin{abstract}
The analysis of gravitational wave data may require greater accuracy than is afforded by the adiabatic approximation to the trajectory of and field produced by a particle moving in curved spacetime. Higher accuracy is available with a two-timescale approach using as an expansion parameter the ratio of orbital time to radiation reaction time. To avoid apparent divergences at large distances, the details of the method are important, especially the choice of the foliation, the spacetime surfaces on which the orbital elements are taken to be constant. Here we apply the two-timescale approach to a simple linear model to demonstrate the details of the method. In particular we use it to show that a null foliation avoids largedistance divergences in the first-order post-adiabatic approximation, and we argue that this will be true more generally for a null foliation.
\end{abstract}

DOI: 10.1103/PhysRevD.77.064001

PACS numbers: 04.25.Nx

\section{INTRODUCTION}

A major target of gravitational wave detectors, such as LIGO, but especially LISA [1], is radiation from extrememass-ratio inspirals (hereafter EMRIs), compact objects, approximated as point particles, orbiting in the field of much larger gravitating centers, typically Kerr black holes. In the zeroth approximation these particles move through the Kerr background on geodesics characterized by orbital constants $E$ (energy), $L$ (angular momentum), and $K$ (Carter constant), and the gravitational radiation generated by the particle is found with perturbation theory to first order in the EMRI ratio, the ratio of the particle mass to the background mass.

In reality, of course, the orbits cannot be geodesics. The loss of energy and angular momentum to radiation requires that the orbits evolve. The evolution can be understood either as a reaction to the radiation, or as the result of a selfforce, the force on the particle due to the spacetime perturbations created by the particle itself. In either viewpoint, the deviation from a geodesic is proportional to the EMRI mass ratio, and for most EMRI ratios this means that the deviation is small enough to justify what is called the adiabatic approximation: the particle is considered to be moving, at any moment, on a geodesic with orbital "constants" $E, L, K$ that are functions of time as the orbit evolves. More important, the same approximation is made for the gravitational waveform generated by the moving particle: the waveform is to be that from a geodesic orbit characterized by $E, L, K$, with the three constants varying in time.

In this adiabatic viewpoint the understanding of the orbit and of the waves it generates is shifted to the question of

\footnotetext{
*mino@tapir.caltech.edu

†rprice@phys.utb.edu
}

the evolution of the orbital constants. The earliest method for doing this was to balance the loss of $E$ and $L$ to the radiation energy and angular momentum going out to infinity and down the horizon [2]. This method, though physically appealing, is not well justified except for circular orbits. Furthermore, it cannot be used to find the evolution of the Carter constant. The alternative, a calculation of the self-force driving the evolution, suffers both from the need for regularization of the divergent perturbation fields at the particle, and the gauge aspects of the self-force. Some consistency of the balance and the self-force methods has been found, when time averaging of the self-force is used [3], although in a restricted gauge.

Many technical details remain unresolved. In particular, the self-force can appear to be entirely gauge dependent on a short timescale [4]. Indeed, along with the issue of gauge invariance, the most pressing problem in this area is the question of the timescale on which the adiabatic approximation can be made to work. For gravitational wave analysis, what is needed is a description that applies over a large change in the orbital parameters. An outline of such a method has been proposed based on considerations of second order particle perturbation theory [5].

In order to formulate a justifiable scheme for an adiabatic approximation, a two-timescale analysis will be needed in which the adiabatic approximation is only the first step in an expansion of fields in orders of the ratio of a slow to a fast timescale [6]. The mathematical framework for doing this was already inherent in Ref. [7], in which it was shown that a consistent two-timescale approach involves several difficulties. First, the post-adiabatic effect is at the same order as nonlinear effects, and the nonlinear gravitational perturbations can result in both infrared-type and ultraviolet-type divergences that are not easy to regularize. Second, there is a weak violation of the gauge condition for the linear perturbations due to radiation 
reaction. The complications of those issues can obscure technical issues inherent in the basic idea of the twotimescale method itself. In particular, if not carried out in the optimal way the two-timescale method can lead to apparent divergences. This is motivation for what is done in the present paper, the application of the two-timescale approach to a well-understood model: a linear scalar field coupled to a point particle.

We use Minkowski coordinates $\{t, x, y, z\}$ and we denote the spacetime coordinates collectively by Greek indices as $x^{\alpha}(\alpha=t, x, y, z)$, and we use the Einstein summation rule with the flat metric, $\eta_{\alpha \beta}=\operatorname{diag}(-1,1,1,1)$. Where appropriate we switch to spherical spatial $\{r, \theta, \phi\}$ coordinates. When we refer specifically to the coordinate location of the particle we use the tilde, as in $\tilde{x}^{\alpha}$. We denote the spatial coordinates by Roman indices as in $x^{i}(i=x, y, z)$. We adopt geometrized units in which $c=G=1$, so that mass, length and time have the same dimension.

As a simplest toy model for the practical demonstration in this paper, we calculate the scalar field $\Phi$ induced by a point charge $q$ according to the flat spacetime wave equation,

$$
\Phi(x)=\rho(x), \quad \rho(x)=q \int d \tau \delta^{(4)}(x-\tilde{x}(\tau)),
$$

where we use the box-operator as $\square=-\left(\partial_{t}\right)^{2}+\left(\partial_{x}\right)^{2}+$ $\left(\partial_{y}\right)^{2}+\left(\partial_{z}\right)^{2}$. Here $\delta^{(4)}(x)$ is the 4-dimensional Dirac's delta and $\tau$ is the proper time of the orbit.

For simplicity, we assume that the particle motion follows a Newtonian second law for the potential of a central gravitating mass $M$,

$$
\frac{d}{d t} \boldsymbol{v}^{i}=-\frac{M \tilde{x}^{i}}{\tilde{r}^{3}}+a^{i},
$$

where $v^{i}=d \tilde{x}^{i} / d \tilde{t}$ is the ordinary spatial velocity (coordinate change per coordinate time) and $a^{i}$ is the ordinary acceleration (in terms of coordinate time) due to scalar radiation reaction.

One of the challenges of point particle perturbation theory is to extract a finite $a^{i}$ from the scalar field which is divergent along the orbit [8]. But our main interest in this paper is the calculation of the first post-adiabatic term in a two-timescale expansion. For that we shall not need an explicit form of $a^{i}$, and will treat it as a known quantity. As one additional simplification, we will consider in this paper only the case of quasicircular orbits (orbits which are circular except for radiation reaction).

The structure of this paper is as follows. In Sec. II, we calculate the orbital equation including the radiation reaction effect. In Sec. III, we give the adiabatic approximation for the scalar field, the leading order of the adiabatic expansion. Though the result here is familiar, the approach in this section clarifies the geometric meaning of that approximation and, in particular, introduces the idea of the spacetime foliation function for evolving orbital ele- ments. In Sec. IV, we calculate the first post-adiabatic correction of the scalar field. We conclude and summarize in Sec. V, and discuss applications of this new expansion scheme to other problems in relativity. In order to have the main text focus on the central ideas, we have relegated many of the details to a set of appendices.

\section{ORBITAL EVOLUTION WITH RADIATION REACTION}

In the adiabatic expansion orbits are characterized by the orbital elements, the integral constants of the orbital equations in the absence of radiation reaction. The evolution of the orbit due to radiation reaction is then described by the evolution of the orbital elements [9]. This means that the orbital elements become functions of "time," i.e., of some parameter $f$ along the orbit. It is through this dependence that the effects of radiation reaction will appear in the orbits. One of the central issues of this paper is to consider just what time slicing of spacetime is appropriate to the evolution of the orbital elements. That is, how is the orbital parameter $f$ to be promoted to a function of spacetime location $f\left(x^{\alpha}\right)$ ?

We align our coordinates so that the quasicircular orbit is at $\tilde{z}=0$ (Cartesians) or $\tilde{\theta}=\pi / 2$ (sphericals). In order to calculate the orbital evolution (1.2), including the radiation reaction effect, it is convenient to use the orbital energy $e$ and the $z$-component of the angular momentum $\ell$ defined by

$$
e=\frac{1}{2} v^{i} v^{i}-\frac{M}{\tilde{r}}, \quad \ell=\tilde{x} v^{y}-\tilde{y} v^{x} .
$$

These are constants of motion which are conserved along the orbit in the absence of radiation reaction. Because of the radiation reaction, $e$ and $\ell$ are not constant and the evolution equations are

$$
\begin{aligned}
\frac{d}{d f} e & :=\frac{d \tilde{t}}{d f} a^{e}=\frac{d \tilde{t}}{d f} v^{i} a^{i}, \\
\frac{d}{d f} \ell & :=\frac{d \tilde{t}}{d f} a^{\ell}=\frac{d \tilde{t}}{d f}\left(\tilde{x} a^{y}-\tilde{y} a^{x}\right) .
\end{aligned}
$$

Here we have computed the dynamics of the orbits with coordinate time $\tilde{t}$, but have used $d \tilde{t} / d f$, evaluated at the orbit, to infer the influence of the dynamics on the orbital elements. We have also defined two components of radiation-reaction driven acceleration: $a^{e}$ and $a^{\ell}$.

Because radiation reaction effect is weak, we first integrate the orbital equation of motion in the absence of the radiation reaction. The orbital coordinates are written as

$$
\begin{aligned}
\tilde{r}(f) & =r_{C}, \quad \tilde{\theta}(f)=\pi / 2, \\
\tilde{\phi}(f) & =\Omega[\tilde{t}(f)-\tilde{t}(f=0)]+\phi_{C},
\end{aligned}
$$

where $r_{C}$ and $\Omega$ are the orbital principal elements, and $\phi_{C}$ is the orbital positional elements. The function $\tilde{t}(f)$ is 
obtained from the definition of the foliation function, $f\left(\tilde{t}, r_{C}, \pi / 2, \tilde{\phi}(f)\right)=f$. Since the orbit (in the absence of radiation reaction) is circular, $e$ and $\ell$ are related by

$$
\ell=\frac{M}{\sqrt{-2 e}} \text {. }
$$

It has been shown that this circularity relationship is not only correct in the absence of radiation reaction, but holds to all orders in an adiabatic expansion, as long as the radiation reaction is small [10]. From this it follows that the two components of the radiation reaction acceleration always obey

$$
a^{\ell}=-\frac{1}{2} \frac{\ell}{e} a^{e} .
$$

For the circular orbit all kinematical quantities are related. It is convenient to express them all in terms of the central mass $M$ and the orbital speed $v$ :

$$
e=-\frac{1}{2} v^{2}, \quad \ell=\frac{M}{v}, \quad r_{C}=\frac{M}{v^{2}}, \quad \Omega=\frac{v^{3}}{M} .
$$

With these relationships we can regard $v$ as an orbital principal element that governs all other principal elements. The evolution of $v$ itself is

$$
\frac{d}{d f} v=-\frac{1}{v} \frac{d \tilde{t}}{d f} a^{e}=-\frac{v^{2}}{M} \frac{d \tilde{t}}{d f} a^{\ell} .
$$

For the relationships in (2.6) to hold in the presence of radiation reaction, $\Omega$ must be defined to be $\Omega:=d \tilde{\phi} / d \tilde{t}$. According to (2.3), this requires that $\phi_{C}$ evolves according to

$$
\frac{d}{d f} \phi_{C}=-[\tilde{t}(f)-\tilde{t}(f=0)] \frac{d}{d f} \Omega .
$$

Because the evolution of the orbital elements is derived perturbatively after we calculate the scalar field by the adiabatic expansion, we start with the orbit as a function of the orbital elements, and we write the source term of the scalar field equation as $\rho\left(x^{\alpha}\right)=\rho\left(x^{\alpha} \mid C\left(f\left(x^{\alpha}\right)\right)\right)$ where we denote by $C$ the collection of orbital elements.

\section{ADIABATIC EXPANSION—LEADING ORDER}

The adiabatic expansion can be elegantly formulated as a two-timescale expansion: One scale is the dynamical scale of the system denoted by $T_{\mathrm{dyn}}$, such as the orbital period or the wavelength of the scalar field. The other is the radiation reaction scale denoted by $T_{\text {rad }}$, such as the timescale for a change in orbital energy due to radiation reaction. We assume that the radiation reaction scale is much longer than the dynamical scale and we use the dimensionless small value $\mu:=T_{\text {dyn }} / T_{\text {rad }} \ll 1$ as an expansion parameter. That is, we assume that the orbital principal elements, the elements directly related to $v$, evolve slowly on the timescale of the radiation reaction. To clarify the order of the two-timescale expansion, we can use $\mu$ as the expansion index and can replace the $f$-derivatives of $v$ by

$$
\left(\frac{d}{d f}\right)^{n} v \rightarrow \mu^{n}\left(\frac{d}{d f}\right)^{n} v
$$

From (2.6), we have that

$$
\frac{d}{d f} r_{C} \sim \frac{d}{d f} \Omega \sim O(\mu), \quad \frac{d^{2}}{d f^{2}} r_{C} \sim \frac{d^{2}}{d f^{2}} \Omega \sim O\left(\mu^{2}\right),
$$

and, from (2.8), that

$$
\begin{aligned}
\frac{d}{d f} \phi_{C} & =-[\tilde{t}(f)-\tilde{t}(f=0)] \frac{d}{d f} \Omega \sim O(\mu), \\
\frac{d^{2}}{d f^{2}} \phi_{C} & =-\frac{d \tilde{t}}{d f} \frac{d}{d f} \Omega-[\tilde{t}(f)-\tilde{t}(f=0)] \frac{d^{2}}{d f^{2}} \Omega \sim O(\mu) .
\end{aligned}
$$

One may ask whether the first $f$-derivative of the orbital positional element $d \phi_{C} / d f$ may become large for large $\tilde{t}(f)$, invalidating the expansion. However, as we will see in the next section, this is not a problem.

In the adiabatic expansion we regard the scalar field as a function of these orbital elements and we expand the field as

$$
\begin{aligned}
\Phi\left(x \mid C\left(f\left(x^{\alpha}\right)\right)\right)= & \Phi^{(0)}\left(x^{\alpha} \mid C\left(f\left(x^{\alpha}\right)\right)\right) \\
& +\mu \Phi^{(1)}\left(x^{\alpha} \mid C\left(f\left(x^{\alpha}\right)\right)\right) \\
& +\mu^{2} \Phi^{(2)}\left(x^{\alpha} \mid C\left(f\left(x^{\alpha}\right)\right)\right)+\cdots .
\end{aligned}
$$

To see how this expansion is used, we consider the wave operator acting on the scalar field. When the differential operator acts on the orbital elements of the scalar field, it results in a factor $\mu$ according to the scheme of (3.1). To the leading order (i.e., order $\mu^{0}$ ) of the adiabatic expansion, we have the equation for $\Phi^{(0)}$ to be

$$
\square\left[\Phi^{(0)}\left(x^{\alpha} \mid C\right)\right]_{C=C\left(f\left(x^{\alpha}\right)\right)}=\rho\left(x^{\alpha} \mid C\left(f\left(x^{\alpha}\right)\right)\right) .
$$

On the left-hand side of (3.5), the wave operator is considered to act only on the direct dependence on $x^{\alpha}$, not on the dependence implicit in the dependence of $C$ on $f$. In this sense, then, the orbital elements can be considered to be constants. By also treating the $C(f)$ on the right as if they were constants, we interpret the wave equation (3.5) as

$$
\left.\square \Phi^{(0)}(x \mid C)=\rho(x \mid C)\right),
$$

for which we can give the well-known solution for a circular orbit with constant orbital elements, 


$$
\begin{aligned}
\Phi^{(0)}\left(x^{\alpha} \mid C\left(f\left(x^{\alpha}\right)\right)\right)= & q \sqrt{1-r_{C}^{2} \Omega^{2}} \sum_{l m}(-i m \Omega) \\
& \times\left(h_{l}^{(1)}(m \Omega r) j_{l}\left(m \Omega r_{C}\right) \theta\left(r-r_{C}\right)\right. \\
& \left.+j_{l}(m \Omega r) h_{l}^{(1)}\left(m \Omega r_{C}\right) \theta\left(r_{C}-r\right)\right) \\
& \times Y_{l m}(\theta, \phi) Y_{l m}^{*}(\pi / 2,0) e^{-i m\left(\Omega t+\phi_{C}\right)},
\end{aligned}
$$

where the orbital elements are evaluated by $r_{C}=$ $r_{C}\left(f\left(x^{\alpha}\right)\right), \Omega=\Omega\left(f\left(x^{\alpha}\right)\right)$ and $\phi_{C}=\phi_{C}\left(f\left(x^{\alpha}\right)\right)$.

We note that, to this leading order of the adiabatic expansion, the foliation function does not play a crucial role in the practical calculation, but it does play an important role in the meaning of the expansion, in particular, how the evolving elements of the orbit affect the solution at points off the orbit. It tells us that the solution at field point $x^{\alpha}$ is the circular orbit solution for the values of $r_{C}, \Omega$, and $\phi_{C}$ that occur at the "time" $f\left(x^{\alpha}\right)$ on the orbit.

\section{FIRST POST-ADIABATIC FIELD}

In the field equation (3.6), we ignored the derivative acting on the orbital elements and, by the rule (3.1), we found a field $\Phi^{(0)}$ that satisfies (1.1) only to accuracy $O(\mu)$. We now turn to the calculation of the solution to the next order in $\mu$.

Using the orders for the derivatives of the orbital elements (3.2) and (3.3) we can substitute the adiabatic expansion (3.4) into the field equation (1.1), and we can extract the terms of order $\mu$. The details are given in Appendix A, and the result is a field equation for the first-order post-adiabatic field $\Phi^{(1)}$ :

$$
\begin{gathered}
{\left[\square \Phi^{(1)}(x \mid C)\right]_{C=C(f)}=\rho^{(1)}(x \mid C(f)),} \\
\rho^{(1)}(x \mid C(f))=-\sum_{C^{a}=r_{C}, \Omega, \phi_{C}}\left\{2\left[\partial_{\alpha} \partial_{a} \Phi^{(0)}(x \mid C)\right]_{C=C(f)}\right. \\
\left.\times g^{\alpha \beta} \partial_{\beta} f+\partial_{a} \Phi^{(0)}(x \mid C(f)) f\right\}\left[\frac{d C^{a}}{d f}\right]^{(1)} \\
-\partial_{\phi_{C}} \Phi^{(0)}(x \mid C(f)) g^{\alpha \beta} \partial_{\alpha} f \partial_{\beta} f\left[\frac{d^{2} \phi_{C}}{d f^{2}}\right]^{(1)},
\end{gathered}
$$

where the notation $[d C / d f]^{(1)}$ and $\left[d^{2} C / d f^{2}\right]^{(1)}$, means that these derivatives are only to be taken to first order in $\mu$. On the left-hand side of (4.2) the derivatives in the $\square$ operator are understood only to act on the explicit $x^{\alpha}$ dependence, and not on the orbital elements. The firstorder nature of the left-hand side is due to the fact that $\Phi^{(1)}$ itself is defined to be first order in (3.4). The result in (4.2), with the orbital elements treated as effective constants on the left is the scalar version of the post-adiabatic equation first derived in Ref. [7].
The foliation function $f\left(x^{\alpha}\right)$ played only a passive role in the adiabatic approximation of Eqs. (3.5), (3.6), and (3.7). By contrast, here the post-adiabatic terms depend explicitly on the choice of $f\left(x^{\alpha}\right)$. The choice of the foliation function is similar to a gauge choice, in that the value of any physical quantity cannot depend on the choice [11]. As a useful check, the foliation-invariance of the first postadiabatic approximation field $\Phi^{(1)}$ is verified in Appendix B.

Though the choice of foliation function is arbitrary in principle, an inappropriate choice will make the first postadiabatic term $\Phi^{(1)}$ divergent so that a regularization procedure will be needed to extract physical information. In Appendix C, we study the asymptotic behavior of the source for the first post-adiabatic term and we find that, if the foliation is asymptotically null, the first postadiabatic term behaves as $\Phi^{(1)} \rightarrow O(1 / r)$ at large radius.

Another insight on the appropriateness of a null foliation comes from the retarded time Lienard-Wiechert solution [12] to (1.1):

$$
\Phi=-\frac{q}{4 \pi} \frac{1}{\left|\vec{x}-\vec{\xi}\left(t^{\prime}\right)\right|-\dot{\vec{\xi}}\left(t^{\prime}\right) \cdot\left(\vec{x}-\vec{\xi}\left(t^{\prime}\right)\right)}
$$

where $\vec{\xi}(\tau)$ has the components $\tilde{z}^{\alpha}(\tau)$ of the orbit of the source particle, and where $t^{\prime}$ is the retarded time, a solution of

$$
t^{\prime}=t-\left|\vec{x}-\vec{\xi}\left(t^{\prime}\right)\right|
$$

For the evolving equatorial orbital motion described by parameters $\Omega(\tau), r_{C}(\tau)$, and $\phi_{C}(\tau)$, Eq. (4.4) becomes

$$
t^{\prime}=t-\sqrt{r^{2}+r_{C}^{2}-2 r r_{C} \sin \theta \cos \left(\Omega\left(t^{\prime}\right) t^{\prime}+\phi_{c}\left(t^{\prime}\right)-\phi\right)} .
$$

The exact solution of (4.5) for $t^{\prime}$ as a function of $t, r, \theta, \phi$ is a null foliation, and would, in a sense, be the perfect foliation for the problem since with this foliation the solution [i.e., that of (4.3)] is guaranteed to have no apparent singularities except at the source points. Equation (4.5) cannot in general be solved exactly, but for $r \gg r_{C}$, and $|t-r| \gg r_{C}$, the approximate solution is a simpler null foliation $t^{\prime}=t-r$.

For simplicity, as well as from the form of the LienardWiechert solution, we choose the foliation

$$
f\left(x^{\alpha}\right)=t-r .
$$

With this choice, $\square f=-2 / r$ in the second term of (4.2), $g^{\alpha \beta} \partial_{\alpha} f \partial_{\beta} f=0$ in the last term, the time coordinate of the orbit is given as $\tilde{t}(f)=f+r_{C}(f)$, and the evolution of $\phi_{C}$ is given by

$$
\frac{d}{d f} \phi_{C}=-\left[t-r+r_{C}(f)-r_{C}(f=0)\right] \frac{d}{d f} \Omega .
$$

This puts the source term of (4.2) in the form 


$$
\begin{aligned}
\rho^{(1)}\left(x \mid r_{C}, \Omega, \phi_{C}\right)= & 2\left[\left(\partial_{t}+\frac{1}{r} \partial_{r} r\right) \frac{\partial}{\partial C^{a}} \Phi^{(0)}(x \mid C)\right]_{C=C(f)} \\
& \times\left[\frac{d C^{a}}{d f}\right]^{(1)} \\
= & q \sqrt{1-v^{2}} \sum_{l m}(-i m \Omega)^{2}\left\{S_{l m}^{(+)} \theta\left(r-r_{C}\right)\right. \\
& \left.+S_{l m}^{(-)} \theta\left(r_{C}-r\right)+S_{l m}^{(p)} r_{C} \delta\left(r-r_{C}\right)\right\} \\
& \times Y_{l m}(\theta, \phi) Y_{l m}^{*}(\pi / 2,0) \\
& \times e^{-i m\left(\Omega t+\phi_{C}\right)}\left[\frac{d v}{d f}\right]^{(1)},
\end{aligned}
$$

where

$$
\begin{aligned}
& S_{l m}^{(+)}=S_{l m}^{(1) j}\left[h_{l}^{(1)}(z)+\frac{i}{z} \frac{d}{d z} z h_{l}^{(1)}(z)\right]_{z=m \Omega r} \\
& +S_{l m}^{(2) j}\left[2 h_{l}^{(1)}(z)+2 z \frac{d}{d z} h_{l}^{(1)}(z)-i 2 z h_{l}^{(1)}(z)\right. \\
& \left.+i \frac{l(l+1)}{z} h_{l}^{(1)}(z)\right]_{z=m \Omega r} \text {, } \\
& S_{l m}^{(-)}=S_{l m}^{(1) h}\left[j_{l}(z)+\frac{i}{z} \frac{d}{d z} z j_{l}(z)\right]_{z=m \Omega r} \\
& +S_{l m}^{(2) h}\left[2 j_{l}(z)+2 z \frac{d}{d z} j_{l}(z)-i 2 z j_{l}(z)\right. \\
& \left.+i \frac{l(l+1)}{z} j_{l}(z)\right]_{z=m \Omega r}, \\
& S_{l m}^{(p)}=-\frac{2}{m^{2} v^{3}}, \\
& S_{l m}^{(1) k}=\left\{-\frac{v}{1-v^{2}}+\frac{3}{v}+3 i m\left(1-\frac{v^{2}}{v^{2}(f=0)}\right)\right\} k_{l}(m v) \\
& +m\left(\frac{d}{d z} k_{l}(z)\right)_{z=m v}, \\
& S_{l m}^{(2) k}=\frac{3}{v} k_{l}(m v),
\end{aligned}
$$

with $k$ representing either $h^{(1)}$ or $j$.

For the most part, the derivation of (4.8), (4.9), (4.10), (4.11), (4.12), and (4.13) is straightforward, but there is at least one point worth mentioning: The derivative with respect to $\phi_{C}$ entails a factor, shown in (4.7), of $t-r+$ $r_{C}(f)-r_{C}(f=0)$. The leading $t$ will cancel with the $t$ arising from the derivative of $e^{-i m \Omega(f) t}$, with respect to $f$, and the $-r$ is rewritten as $-m \Omega z$, leaving us with

$$
\begin{aligned}
r_{C}(f)-r_{C}(f & =0)=\Omega^{-1} v\left[1-\frac{r_{C}(f=0)}{r_{C}(f)}\right] \\
& =\Omega^{-1} v\left[1-\frac{v^{2}(f)}{v^{2}(f=0)}\right] .
\end{aligned}
$$

Here the relationship $r_{C}=M / v^{2}$, from (2.6), has been used and, as usual, the symbols $\Omega$ and $v$, with no explicit argument are understood to be $\Omega(f)$ and $v(f)$.

For the source in (4.8), (4.9), (4.10), (4.11), (4.12), and (4.13), the solution of (4.1) for $\Phi^{(1)}$ can be found using the standard retarded Green function for the wave operator. This solution is summarized in Appendix D. It should be noted that the source term above has a discrete spectrum and contains only Fourier components with $\omega=m \Omega$ and that each $(l m)$-mode of $S_{l m}^{k}$ contributes only to the $(\mathrm{lm})$-mode of $\Phi^{(0)}$. This is due to our spherically symmetric choice of the foliation in (4.6). With this choice, no explicit angular dependence is introduced into the differential operators. The separation of Fourier modes stops when we come to the explicit evaluation of $[d v / d f]^{(1)}$. This is a nonlinear step that mixes the contributions from all Fourier modes.

\section{SUMMARY AND DISCUSSION}

We have applied the two-timescale method to the simple well-understood problem of a slowly evolving quasicircular orbit of a scalar charged particle in a central potential. The question motivating this work was that of the justification, and applicability of the adiabatic approximation and its extension. We have focused on the manner in which the slowly changing orbital elements of the trajectory, the radius $r_{C}$, angular velocity $\Omega$, and phase constant $\phi_{C}$ are to be promoted to spacetime fields through the choice of some foliation function $f\left(x^{\alpha}\right)$. We have shown that this choice, which plays only a passive role in the lowest-order adiabatic expansion, is crucial for the post-adiabatic fields. In particular, we show that the first-order adiabatic field $\Phi^{(1)}$ is well behaved as $r \rightarrow \infty$ if $f$ is chosen to be $t-r$, but is not well behaved for a general choice of foliation function, in particular, for the acausal choice $f=t$, which has the orbital elements change throughout spacetime according to coordinate time.

An advantage of our simple model is that we have an alternative approach to the description of the field, the Lienard-Wiechert solution. We can get insight into the pathology of the wrong foliation by considering the $r \gg$ $r_{C}$ form of the Lienard-Wiechert solution in (4.3)

$$
\Phi=-\frac{q}{4 \pi r} \frac{1}{1-\dot{\vec{\xi}}\left(t^{\prime}\right) \cdot \vec{x} / r}
$$

We have already argued, on the basis of the retardation equations (4.4) or (4.5), that $f=t-r$ is the correct large $r$, foliation function, but now we can consider what would happen for the foliation choice $t^{\prime}=t$. In this case, at large $r$, the value of $\dot{\vec{\xi}}\left(t^{\prime}=t\right)$ would differ from the correct value $\dot{\vec{\xi}}\left(t^{\prime}=t-r\right)$, by an amount that is proportional to $r$ and thus would constitute a contribution to the field that does not fall off as $1 / r$. 
It should be noted that the Lienard-Wiechert solution is itself a demonstration that for our simple problem the adiabatic approximation can be made to be exact over an arbitrarily long timescale. In (4.3) one needs only to take the exact solution for $t^{\prime}$ as the foliation and to describe the motion $\vec{\xi}(\tau)$ in terms of evolving orbital constants, a description that can always be given if enough constants are included. From this point of view, adiabatic expansions of different orders represent various accuracies with which the foliation function $f$ is equal to the $t^{\prime}$ that solves (4.4). In our equatorial orbit problem, the usual lowest order adiabatic approximation [say that of (3.7)] corresponds to the following procedure: One fixes the orbital constants $\Omega, r_{C}$ and $\phi_{C}$, then solves symbolically for $t^{\prime}\left(x^{\alpha}\right)$ parametrized by these constants and writes the Lienard-Wiechert solution in terms of that solution for $t^{\prime}$. In this solution, one then promotes the constants to functions of whatever foliation is chosen.

The Lienard-Wiechert solution, of course, applies only to problems involving linear fields obeying the flat spacetime wave equation. The two-timescale approach, in principle, applies much more generally. But at least four important questions remain about the two-timescale approach. The first is whether it converges for our simple problem (or similar linear problems). We feel that the existence and form of the Lienard-Wiechert solution all but guarantees that the post-adiabatic expansion, if done correctly, must converge. The caveat here, that the expansion must be done correctly, points to the need for the correct choice of foliation. A general argument based on (5.1), and the details of the post-adiabatic equation in (A9) and its higher order extensions, both point to the choice $f=t-r$ being adequate to all orders.

The second important question about the expansion is whether it converges for fields in curved spacetimes, such as black hole spacetimes. The issue is the choice of the foliation around the black hole horizon and the convergence of the post-adiabatic expansion. The curved-space generalization of the Lienard-Wiechert solution is obtained by the formal Hadamard series expansion using the bitensor formalism [13]. On the basis of this we suspect that the post-adiabatic expansion must converge with the choice of null foliation to the future horizon, but this is a question that deserves further study.

The third important question about the expansion is the issue of the gauge condition when we apply this expansion to the gravitational perturbation problem [7]. In Sec. III, we found that the first $f$-derivative of the positional element (3.3) could be large for large $\tilde{t}(f)$. However, as we see from (4.8), this does not invalidate the post-adiabatic expansion in our simple scalar model. This is not the case for gravitational perturbations and it is necessary to choose an appropriate gauge condition for the post-adiabatic expansion so that gravitational perturbations become valid on a longer time scale [7].
The last important question about the expansion is whether it converges for more realistic problems in relativity, especially nonlinear problems including the dynamics of geometry. Here we feel that the issue of divergences at large- $r$ will probably be similar or identical to that for the linear problems, since the fields are weak at large $r$. The issue of the post-adiabatic expansion near strong field sources is one that will have to await the exploration of a nonlinear model. A point deserving particular attention is that the dynamical horizon may complicate the analysis and the convergence due to the time evolution of the black hole mass. To get some insights about nonlinear issues, we hope to apply the two-timescale method to is a post-helical solution based on a helical nonlinear problem, such as that in the post-Minkowski solution of Beetle et al. [14]

\section{ACKNOWLEDGMENTS}

We gratefully acknowledge support for this work under NSF grants PHY-0601459 and PHY-0554367, by the Brinson Foundation, and by the Center for Gravitational Wave Astronomy. We thank Éanna Flanagan for helpful suggestions.

\section{APPENDIX A: ADIABATIC EXPANSION OF THE WAVE OPERATOR}

In this appendix, we consider the two-timescale expansion of the wave operator. For the application of the twotimescale expansion, we regard the scalar field $\Phi$ as a function of the spacetime coordinates $\left\{x^{\alpha}\right\}$ and of the orbital elements, $\Omega, r_{C}$ and $\phi_{C}$, collectively denoted $C^{a}$. In addition to its direct dependence on the spacetime coordinates, the scalar field has an additional dependence through the foliation function $f\left(x^{\alpha}\right)$ :

$$
\Phi=\Phi(x \mid C(f)) .
$$

As explained in (3.1), in the two-timescale expansion we assume that the derivative of the orbital principal elements is small, and, in particular, that

$$
\frac{d^{n}}{d f^{n}} v \sim O\left(\mu^{n}\right),
$$

where $\mu$ is the ratio of dynamic to radiation-reaction timescales. The derivative of the orbital positional elements is obtained from the orbital equation of motion.

It is crucial to deal separately with the coordinate derivative of the scalar field with fixed $C^{a}$ and with the coordinate derivative of the scalar field due to the spacetime dependence implicit in $C^{a}\left(f\left(x^{\alpha}\right)\right)$. For clarity of the notation, we use

$$
\left[\partial_{\alpha} \Phi(x \mid C)\right]_{C=C(f)},
$$

to denote the derivative with respect to the coordinates with the orbital elements $C^{a}$ fixed. We also use 


$$
\partial_{a} \Phi:=\left[\frac{\partial}{\partial C^{a}} \Phi(x \mid C)\right]_{C=C(f)}
$$

to denote the derivative with respect to the orbital elements $C^{a}$.

With these notations, the partial derivative of the scalar field is written as

$$
\partial_{\alpha} \Phi(x \mid C(f))=\left[\partial_{\alpha} \Phi\right]_{C=C(f)}+\partial_{a} \Phi \frac{d C^{a}}{d f} \partial_{\alpha} f
$$

The second partial derivative becomes

$$
\begin{aligned}
\partial_{\alpha} \partial_{\beta} \Phi(x \mid C(f))= & {\left[\partial_{\alpha} \partial_{\beta} \Phi\right]_{C=C(f)}+\left[\partial_{a} \partial_{\alpha} \Phi\right]_{C=C(f)} } \\
& \times \frac{d C^{a}}{d f} \partial_{\beta} f+\left[\partial_{a} \partial_{\beta} \Phi\right]_{C=C(f)} \frac{d C^{a}}{d f} \partial_{\alpha} f \\
& +\partial_{a} \partial_{b} \Phi \frac{d C^{a}}{d f} \frac{d C^{b}}{d f} \partial_{\alpha} f \partial_{\beta} f \\
& +\partial_{a} \Phi\left(\frac{d C^{a}}{d f} \partial_{\alpha} \partial_{\beta} f+\frac{d^{2} C^{a}}{d f^{2}} \partial_{\alpha} f \partial_{\beta} f\right)
\end{aligned}
$$

where we use the fact that the coordinate derivative with the fixed orbital elements commute with the derivative with respect to the orbital elements, i.e., $\left[\partial_{a} \partial_{\alpha} \Phi\right]_{C=C(f)}=$ $\left[\partial_{\alpha} \partial_{a} \Phi\right]_{C=C(f)}$.

Using the results above, we have that in general spacetime coordinates the wave operator acting on the scalar field is

$$
\begin{aligned}
\square \Phi= & {[\square \Phi]_{C=C(f)}+g^{\alpha \beta}\left(2\left[\partial_{\alpha} \partial_{a} \Phi\right]_{C=C(f)} \partial_{\beta} f\right.} \\
& \left.+\partial_{a} \Phi \partial_{\alpha} \partial_{\beta} f-\Gamma_{\alpha \beta}^{\gamma} \partial_{a} \Phi \partial_{\gamma} f\right) \frac{d C^{a}}{d f} \\
& +\left(\partial_{a} \partial_{b} \Phi \frac{d C^{a}}{d f} \frac{d C^{b}}{d f}+\partial_{a} \Phi \frac{d^{2} C^{a}}{d f^{2}}\right) g^{\alpha \beta} \partial_{\alpha} f \partial_{\beta} f
\end{aligned}
$$

where $\Gamma_{\alpha \beta}^{\gamma}$ is the Christoffel symbol.

In applying (A7) to find adiabatic fields of any order, one must count the order by adding the index $n$ of the field $\Phi^{(n)}$ to the number of $C^{a}$ derivatives, keeping in mind that $d^{2} C^{a} / d f^{2}$ can be first or second order. Thus, for example, $g^{\alpha \beta} \partial_{\alpha} \partial_{a} \Phi^{(2)}\left(d C^{a} / d f\right) f_{, \beta}$ is third order and contributes only to the source for $\square \Phi^{(3)}$, while $\partial_{\alpha} \partial_{a} \Phi^{(2)}\left(d^{2} C^{a} / d f^{2}\right) g^{\alpha \beta} f_{, \alpha} f_{, \beta}$ is a source for both $\square \Phi^{(3)}$ and $\square \Phi^{(4)}$.

To first order, the source that result from (A7) is that in (4.2). To second order, the result is

$$
\left[\square \Phi^{(2)}(x \mid C)\right]_{C=C(f)}=\rho^{(2)}(x \mid C(f)),
$$

$$
\begin{aligned}
\rho^{(2)}(x \mid C(f))= & -\left(\partial_{a} \partial_{b} \Phi^{(0)}\left[\frac{d C^{a}}{d f}\right]^{(1)}\left[\frac{d C^{b}}{d f}\right]^{(1)}\right. \\
& \left.+\partial_{a} \Phi^{(0)}\left[\frac{d^{2} C^{a}}{d f^{2}}\right]^{(2)}\right) g^{\alpha \beta} \partial_{\alpha} f \partial_{\beta} f \\
& -\left\{g^{\alpha \beta} 2\left[\partial_{\alpha} \partial_{a} \Phi^{(1)}(x \mid C)\right]_{C=C(f)} \partial_{\beta} f\right. \\
& \left.+\partial_{a} \Phi^{(1)}(x \mid C(f)) \square f\right\}\left[\frac{d C^{a}}{d f}\right]^{(1)} \\
& -\partial_{a} \Phi^{(1)}\left[\frac{d^{2} C^{a}}{d f^{2}}\right]^{(1)} g^{\alpha \beta} \partial_{\alpha} f \partial_{\beta} f .
\end{aligned}
$$

It should be noted that for null foliations the factor $g^{\alpha \beta} f_{\alpha} f_{\beta}$ vanishes, simplifying the results.

\section{APPENDIX B: INVARIANCE OF THE FIRST POST-ADIABATIC EXPANSION}

Our adiabatic expansion uses a foliation function that is arbitrary in principle. If the result of the adiabatic expansion depends on the choice of this foliation, it means there must be a physical condition to determine the foliation. Otherwise, the adiabatic expansion would not have physical predictability. In this appendix, we shall prove that the field found by the adiabatic expansion does not depend on the choice of the foliation to the first post-adiabatic expansion, and therefore, at least to this order, does have physical predictability.

We consider an infinitesimal change in the foliation function

$$
f\left(x^{\alpha}\right) \rightarrow f\left(x^{\alpha}\right)+\delta f\left(x^{\alpha}\right)
$$

and we show here that the first post-adiabatic field $\Phi^{(1)}$ does not change to first order in $\delta f$.

There are two constraints on our choice of $f$, and hence on $\delta f$. First, we recall the role of the foliation function. The orbital elements $C$, that are defined by the evolving orbit are promoted to spacetime functions by making them functions $C\left(f\left(x^{\alpha}\right)\right)$. We choose to leave constant the value of $f$ on every point of the orbit. (To do otherwise would not really involve a change in the foliation, but rather would be a change in the parametrization of the orbit that would change the functional forms of the $C(f)$ ). This gives us the constraint

$$
\delta f\left(x^{\alpha}\right)=0, \quad \text { for } x^{\alpha} \text { on the orbit. }
$$

Our second constraint is that we choose $\delta f$ to behave at infinity so that

$$
\frac{\partial \delta f}{\partial x^{\alpha}} \rightarrow \text { constant, } \quad \text { as } r \rightarrow \infty
$$

The reason for this, to be clarified below, is connected to the large- $r$ boundary conditions that determine the solution for $\Phi^{(1)}$. 
The differential change $\delta f$ affects the field $\Phi=\Phi^{(0)}+$ $\Phi^{(1)}+\cdots$ in two ways. First, at a given spacetime point $x^{\alpha}$ the field formally denoted as $\Phi=\Phi^{(0)}$ will have a change of order $\mu$ according to

$$
\begin{aligned}
\Phi^{(0)}(x \mid C(f)) & \rightarrow \Phi^{(0)}(x \mid C(f+\delta f)) \\
& =\Phi^{(0)}(x \mid C(f))+\delta \Phi^{(0)}(x \mid C(f)),
\end{aligned}
$$

where

$$
\delta \Phi^{(0)}(x \mid C(f))=\partial_{a} \Phi^{(0)} \frac{d C^{a}}{d f} \delta f .
$$

Because $d C^{a} / d f$ is $O(\mu)$ by the ansatz of the twotimescale expansion (A2), $\delta \Phi^{(0)}$ is regarded as the part of the first post-adiabatic term. We note that

$$
\begin{aligned}
{\left[\square \delta \Phi^{(0)}\right]_{C=C(f)}=} & \square\left[\partial_{a} \Phi^{(0)} \frac{d C^{a}}{d f} \delta f\right] \\
= & {\left[2 g^{\alpha \beta} \partial_{a} \partial_{\alpha} \Phi^{(0)} \delta_{\beta} f\right.} \\
& \left.+\partial_{a} \Phi^{(0)} \square \delta f\right] \frac{d C^{a}}{d f} .
\end{aligned}
$$

The term $\partial_{a} \square \Phi^{(0)} \delta f\left(d C^{a} / d f\right)$ has been omitted, since $\square \Phi^{(0)}$ vanishes except on the orbit, where, by constraint (B2) the factor $\delta f$ vanishes.

To find the change in the formal post-adiabatic term $\Phi^{(1)}$ we consider the transformation of $\rho^{(1)}$

$$
\rho^{(1)} \rightarrow \rho^{(1)}+\delta \rho^{(1)} .
$$

We are interested in $\delta \rho^{(1)}$, the change, to first order in $\mu$, to the right-hand side of (4.8) induced by $\delta f$. It is crucial to see that $\delta \rho^{(1)}$ does not come from changes in $\Phi^{(0)}$ nor to $\left[d C^{a} / d f\right]^{(0)}$ in that right-hand side; such changes would be post-adiabatic terms higher order in $\mu$. To first order in $\mu$ the only changes induced by the change (B1) are those in

$$
\begin{aligned}
\delta \rho^{(1)}= & -\left\{2\left[\partial_{\alpha} \partial_{a} \Phi^{(0)}(x \mid C)\right]_{C=C(f)} g^{\alpha \beta} \partial_{\beta} \delta f\right. \\
& \left.+\partial_{a} \Phi^{(0)}(x \mid C(f)) \square \delta f\right\}\left[\frac{d C^{a}}{d f}\right]^{(0)} .
\end{aligned}
$$

In principle, we can find $\delta \Phi^{(1)}$ from

$$
\left[\square \delta \Phi^{(1)}\right]_{C=C(f)}=\delta \rho^{(1)} .
$$

Since the right-hand sides of (B6) and (B8) add to zero, we have

$$
\left[\square\left(\delta \Phi^{(1)}+\delta \Phi^{(0)}\right)\right]_{C=C(f)}=0,
$$

so the wave equation is sourceless for the change $\delta \Phi$ of the scalar field, to first adiabatic order.

In order to conclude that the change $\delta \Phi$ is zero to first order, we need to rule out "free wave" solutions of (B10). This can be accomplished by imposing the constraint in (B3). If some such constraint is not imposed then nonzero solutions for $\delta \Phi$ exist, and a change in the foliation function appears to make a change, to first adiabatic order, in the solution for $\Phi$. But the change in the foliation function would entail a change from a solution that is causal, i.e., one that has only outgoing waves, to one that is not causal. In the adiabatic expansion we use only causal Green functions to solve for the various orders of $\Phi^{(n)}$.

\section{APPENDIX C: ASYMPTOTIC BEHAVIOR OF THE SOURCE TERM FOR THE FIRST POST-ADIABATIC TERM}

In this appendix, we consider a general spherical foliation defined as

$$
f=f(t, r),
$$

and we investigate the asymptotic behavior of the source term for the first post-adiabatic field with this general foliation function. We use the asymptotic form of the adiabatic scalar field written as

$$
\begin{aligned}
\Phi^{(0)}(x \mid C(f))= & \frac{1}{r} \sum_{l m ; n=0,1,2, \cdot} A_{l m}^{(n)}(C(f)) Y_{\ell m}(\theta, \phi) \\
& \times \frac{1}{r^{n}} e^{-i m\left(\Omega(f)(t-r)+\phi_{C}(f)\right)} .
\end{aligned}
$$

[See (3.7) for the full expression.]

The source term for the first post-adiabatic term, for the spherically symmetric foliation is

$$
\begin{aligned}
\rho^{(1)}(x ; C)= & \sum_{C^{a}=\Omega, \phi_{C}}\left\{\left[\left(2 \partial_{t} f \partial_{t}-2 \partial_{r} f \partial_{r}-\frac{2}{r} \partial_{r} f\right.\right.\right. \\
& \left.\left.+\partial_{t}^{2} f-\partial_{r}^{2} f\right) \frac{\partial}{\partial C^{a}} \Phi^{(0)}(x, C)\right]\left[\frac{d C^{a}}{d f}\right]^{(1)} \\
& \left.+\left(\left(\partial_{t} f\right)^{2}-\left(\partial_{t} f\right)^{2}\right) \frac{\partial}{\partial C^{a}} \Phi^{(0)}(x, C)\left[\frac{d^{2} C^{a}}{d f^{2}}\right]^{(1)}\right\} .
\end{aligned}
$$

The regular behavior of $\Phi$, and hence of the first postadiabatic term $\Phi^{(1)}$ is $\sim O\left(r^{-1}\right)$ at large $r$. For this to be the case, the first-order source term $\rho^{(1)}$ must fall off at large $r$ as $O\left(r^{-3}\right)$, and this condition constrains the foliation function. To order $1 / r$, in the expression above

$$
2 \partial_{t} f \partial_{t}-2 \partial_{r} f \partial_{r}=-2 i m\left(\partial_{t} f+\partial_{r} f\right) .
$$

The function $\partial_{a} C^{a} \Phi^{(0)}(x, C)$ falls off as $1 / r$, hence $\rho^{(1)}$ is asymptotically $1 / r$ unless the leading terms vanish, that is unless

$$
0=\partial_{t} f+\partial_{r} f=\partial_{t}^{2} f-\partial_{r}^{2} f,
$$

which indicates that the foliation surface must asymptotically be a future null cone. By these conditions, we have the foliation function written as

$$
f(t, r) \rightarrow f(t-r) \quad \text { at } r \rightarrow \infty .
$$

If we put $f=t-r$ in (C3) we get 


$$
\begin{aligned}
\rho^{(1)}(x ; C)= & 2 \sum_{C^{a}=\Omega, \phi_{C}}\left[\left(\partial_{t}+\partial_{r}+\frac{1}{r}\right) \frac{\partial}{\partial C^{a}} \Phi^{(0)}(x, C)\right] \\
& \times\left[\frac{d C^{a}}{d f}\right]^{(1)} .
\end{aligned}
$$

Since

$$
\begin{aligned}
\partial_{a} C^{a} \Phi^{(0)}(x, C)= & \frac{1}{r} e^{-i m\left(\Omega(f)(t-r)+\phi_{C}(f)\right)}\left[\mathcal{A}\left(C^{a}, \theta, \phi\right)\right. \\
& \left.+\frac{B\left(C^{a}, \theta, \phi\right)}{r}+\cdots\right],
\end{aligned}
$$

we have that $\rho^{(1)}(x ; C)$ falls off as $r^{-3}$, as it must.

\section{APPENDIX D: INTEGRAL FORMULA FOR THE FIRST POST-ADIABATIC TERM}

We summarize the integral formula for the field equation under the outgoing-wave boundary condition. We consider the scalar wave equation

$$
\square F\left(x^{\alpha}\right)=S\left(x^{\alpha}\right),
$$

for flat spacetime, i.e., $\square=-\partial_{t}^{2}+\partial_{x}^{2}+\partial_{y}^{2}+\partial_{z}^{2}$. In spherical coordinates, it is convenient to use the decomposition

$$
\begin{aligned}
& F(x)=\sum_{\operatorname{lm} \omega} F_{l m \omega}(r) Y_{l m}(\theta, \phi) e^{-i \omega t}, \\
& S(x)=\sum_{l m \omega} S_{l m \omega}(r) Y_{l m}(\theta, \phi) e^{-i \omega t} .
\end{aligned}
$$

A formal solution of the radial mode functions is obtained by the Green's method as

$$
F_{l m \omega}(r)=\int d r^{\prime} g_{\operatorname{lm} \omega}\left(r, r^{\prime}\right) S_{l m \omega}(r),
$$

$$
\begin{aligned}
g_{l m \omega}\left(r, r^{\prime}\right)= & -i \omega r^{\prime 2}\left(h_{l}^{(1)}(\omega r) j_{l}\left(\omega r^{\prime}\right) \theta\left(r-r^{\prime}\right)\right. \\
& \left.+j_{l}(\omega r) h_{l}^{(1)}\left(\omega r^{\prime}\right) \theta\left(r^{\prime}-r\right)\right),
\end{aligned}
$$

where $\theta(x)$ is the step function, i.e. $\theta(x)=1$ when $x>0$ and $\theta(x)=0$ otherwise.

For the calculation of the first post-adiabatic term, we write the source term schematically as

$$
\begin{aligned}
S_{l m \omega}(r)= & s_{l m \omega}^{p} r_{C} \delta\left(r-r_{C}\right)+\left\{s_{l m \omega}^{h(1)}\left[h_{l}^{(1)}(z)+\frac{1}{z} \frac{d}{d z} z h_{l}^{(1) \prime}(z)\right]_{z=\omega r}+s_{l m \omega}^{h(2)}\left[h_{l}^{(1)}(z)+z \frac{d}{d z} h_{l}^{(1)}(z)\right]_{z=\omega r}\right. \\
& \left.+s_{l m \omega}^{h(3)}\left[-i 2 z h_{l}^{(1)}(z)+i \frac{l(l+1)}{z} h_{l}^{(1)}(z)\right]_{z=\omega r}\right\} \theta\left(r-r_{C}\right)+\left\{s_{l m \omega}^{j(1)}\left[j_{l}(z)+\frac{1}{z} \frac{d}{d z} z j_{l}(z)\right]_{z=\omega r}\right. \\
& \left.+s_{l m \omega}^{j(2)}\left[j_{l}(z)+z \frac{d}{d z} j_{l}(z)\right]_{z=\omega r}+s_{l m \omega}^{j(3)}\left[-i 2 z j_{l}(z)+i \frac{l(l+1)}{z} j_{l}(z)\right]_{z=\omega r}\right\} \theta\left(r_{C}-r\right),
\end{aligned}
$$

where $s_{\operatorname{lm} \omega}^{h / j(1 / 2 / 3)}$ does not depend on $r$.

The radial mode function induced by the first term on the right-hand side of (D5) is obtained in a trivial manner as

$$
\begin{aligned}
F_{l m \omega}^{p}(r)= & \int d r^{\prime} g_{l m \omega}\left(r, r^{\prime}\right) s_{l m \omega}^{p} r_{C} \delta\left(r^{\prime}-r_{C}\right) \\
= & -i \omega r_{C}^{3} s_{l m \omega}^{p}\left(h_{l}^{(1)}(\omega r) j_{l}\left(\omega r_{C}\right) \theta\left(r-r_{C}\right)\right. \\
& \left.+j_{l}(\omega r) h_{l}^{(1)}\left(\omega r_{C}\right) \theta\left(r_{C}-r\right)\right) .
\end{aligned}
$$

The radial mode functions induced by the second, third, fifth, and sixth terms on the right-hand side of (D5) are obtained in a closed form as

$$
\begin{aligned}
F_{l m \omega}^{h(1)}(r)= & \int d r^{\prime} g_{l m \omega}\left(r, r^{\prime}\right) s_{l m \omega}^{h(1)}\left[h_{l}^{(1)}(z)\right. \\
& \left.+\frac{i}{z} \frac{d}{d z} z h_{l}^{(1)}(z)\right]_{z=\omega r^{\prime}} \theta\left(r^{\prime}-r_{C}\right) \\
= & -\frac{i}{\omega^{2}} s_{l m \omega}^{h(1)}\left[\left\{\frac{i}{4} z\left(h_{l+1}^{(1)}(z)-h_{l-1}^{(1)}(z)\right)\right.\right. \\
& \left.-\frac{1}{2}\left(z-z_{C}\right) h_{l}^{(1)}(z)+f_{l m \omega}^{h(1)+} h_{l}^{(1)}(z)\right\} \theta\left(r-r_{C}\right) \\
& \left.+f_{l m \omega}^{h(1)-} j_{l}(z) \theta\left(r_{C}-r\right)\right],
\end{aligned}
$$

$$
\begin{aligned}
f_{l m \omega}^{h(1)+}=- & \frac{z_{C}^{3}}{4}\left(2 j_{l}\left(z_{C}\right) h_{l}^{(1)}\left(z_{C}\right)-j_{l-1}\left(z_{C}\right) h_{l+1}^{(1)}\left(z_{C}\right)\right. \\
& \left.-j_{l+1}\left(z_{C}\right) h_{l-1}^{(1)}\left(z_{C}\right)\right)-\frac{i}{2} z_{C}^{2} j_{l}\left(z_{C}\right) h_{l}^{(1)}\left(z_{C}\right), \\
f_{l m \omega}^{h(1)-}= & -\frac{z_{C}^{3}}{2}\left(h_{l}^{(1) 2}\left(z_{C}\right)-h_{l-1}^{(1)}\left(z_{C}\right) h_{l+1}^{(1)}\left(z_{C}\right)\right) \\
& -\frac{i}{2} z_{C}^{2} h_{l}^{(1) 2}\left(z_{C}\right)
\end{aligned}
$$

$$
\begin{aligned}
F_{l m \omega}^{h(2)}(r)= & \int d r^{\prime} g_{l m \omega}\left(r, r^{\prime}\right) s_{l m \omega}^{h(2)}\left[h_{l}^{(1)}(z)\right. \\
& \left.+z \frac{d}{d z} h_{l}^{(1)}(z)\right]_{z=\omega r^{\prime}} \theta\left(r^{\prime}-r_{C}\right) \\
= & -\frac{i}{\omega^{2}} s_{l m \omega}^{h(2)}\left[\left\{-\frac{i}{8} z\left(h_{l+1}^{(1)}(z)-h_{l-1}^{(1)}(z)\right)\right.\right. \\
& \left.+\frac{i}{4}\left(z^{2}-z_{C}^{2}\right) h_{l}^{(1)}(z)+f_{l m \omega}^{h(2)+} h_{l}^{(1)}(z)\right\} \theta\left(r-r_{C}\right) \\
& \left.+f_{l m \omega}^{h(2)-} j_{l}(z) \theta\left(r_{C}-r\right)\right],
\end{aligned}
$$




$$
\begin{aligned}
& f_{l m \omega}^{h(2)+}=-\frac{z_{C}^{3}}{8}\left(2 j_{l}\left(z_{C}\right) h_{l}^{(1)}\left(z_{C}\right)+j_{l-1}\left(z_{C}\right) h_{l+1}^{(1)}\left(z_{C}\right)\right. \\
& \left.+j_{l+1}\left(z_{C}\right) h_{l-1}^{(1)}\left(z_{C}\right)\right) \text {, } \\
& f_{l m \omega}^{h(2)-}=-\frac{z_{C}^{3}}{4}\left(h_{l}^{(1) 2}\left(z_{C}\right)+h_{l-1}^{(1)}\left(z_{C}\right) h_{l+1}^{(1)}\left(z_{C}\right)\right), \\
& F_{l m \omega}^{j(1)}(r)=\int d r^{\prime} g_{\operatorname{lm} \omega}\left(r, r^{\prime}\right) s_{\operatorname{lm} \omega}^{j(1)}\left[j_{l}(z)\right. \\
& \left.+\frac{i}{z} \frac{d}{d z} z j_{l}(z)\right]_{z=\omega r^{\prime}} \theta\left(r_{C}-r^{\prime}\right) \\
& =-\frac{i}{\omega^{2}} s_{l m \omega}^{j(1)}\left[f_{l m \omega}^{j(1)+} h_{l}(z) \theta\left(r-r_{C}\right)\right. \\
& +\left\{\frac{i}{4} z\left(j_{l+1}(z)-j_{l-1}(z)\right)-\frac{1}{2}\left(z-z_{C}\right) j_{l}(z)\right. \\
& \left.\left.+f_{l m \omega}^{j(1)-} j_{l}(z)\right\} \theta\left(r_{C}-r\right)\right] \text {, } \\
& f_{l m \omega}^{j(1)+}=\frac{z_{C}^{3}}{2}\left(j_{l}^{2}\left(z_{C}\right)-j_{l-1}\left(z_{C}\right) j_{l+1}\left(z_{C}\right)\right)+\frac{i}{2} z_{C}^{2} j_{l}^{2}\left(z_{C}\right), \\
& f_{l m \omega}^{j(1)-}=\frac{z_{C}^{3}}{4}\left(2 j_{l}\left(z_{C}\right) h_{l}^{(1)}\left(z_{C}\right)-j_{l-1}\left(z_{C}\right) h_{l+1}^{(1)}\left(z_{C}\right)\right. \\
& \left.-j_{l+1}\left(z_{C}\right) h_{l-1}^{(1)}\left(z_{C}\right)\right)+\frac{i}{2} z_{C}^{2} j_{l}\left(z_{C}\right) h_{l}^{(1)}\left(z_{C}\right), \\
& F_{l m \omega}^{j(2)}(r)=\int d r^{\prime} g_{l m \omega}\left(r, r^{\prime}\right) s_{\operatorname{lm} \omega}^{j(2)}\left[j_{l}(z)\right. \\
& \left.+z \frac{d}{d z} j_{l}(z)\right]_{z=\omega r^{\prime}} \theta\left(r_{C}-r^{\prime}\right) \\
& =-\frac{i}{\omega^{2}} s_{l m \omega}^{j(2)}\left[f_{l m \omega}^{j(2)+} h_{l}(z) \theta\left(r-r_{C}\right)\right. \\
& +\left\{-\frac{i}{8} z\left(j_{l+1}(z)-j_{l-1}(z)\right)+\frac{i}{4}\left(z^{2}-z_{C}^{2}\right) j_{l}(z)\right. \\
& \left.\left.+f_{l m \omega}^{j(2)-} j_{l}(z)\right\} \theta\left(r_{C}-r\right)\right] \text {, } \\
& f_{l m \omega}^{j(2)+}=\frac{z_{C}^{3}}{4}\left(j_{l}^{2}\left(z_{C}\right)+j_{l-1}\left(z_{C}\right) j_{l+1}\left(z_{C}\right)\right), \\
& f_{l m \omega}^{j(2)-}=\frac{z_{C}^{3}}{8}\left(2 j_{l}\left(z_{C}\right) h_{l}^{(1)}\left(z_{C}\right)+j_{l-1}\left(z_{C}\right) h_{l+1}^{(1)}\left(z_{C}\right)\right. \\
& \left.+j_{l+1}\left(z_{C}\right) h_{l-1}^{(1)}\left(z_{C}\right)\right) \text {, }
\end{aligned}
$$

where we use $z=\omega r$ and $z_{C}=\omega r_{C}$.

Unlike the previous terms, the radial mode functions induced by the fourth and seventh terms on the righthand side of (D5) cannot be obtained in closed form. For them, it is necessary to obtain the following indefinite integrals,

$$
\begin{aligned}
D_{l}^{(1) k k^{*}} & =\int d z z k_{l}(z) k_{l}^{*}(z), \\
D_{l}^{(2) k k^{*}} & =\int d z z^{3} k_{l}(z) k_{l}^{*}(z),
\end{aligned}
$$

where $k$ and $k^{*}$ represent either $j$ or $h^{(1)}$. For finite $l$, these integrals can be obtained from the recurrence formula

$$
\begin{aligned}
D_{l}^{(1) k k^{*}}= & D_{l-1}^{(1) k k^{*}}-\frac{1}{2 l} z^{2}\left(k_{l}(z) k_{l}^{*}(z)+k_{l-1}(z) k_{l-1}^{*}(z)\right), \\
D_{l}^{(2) k k^{*}}= & \frac{l+1}{l-1} D_{l-1}^{(2) k k^{*}}-\frac{1}{2(l-1)} z^{4}\left(k_{l}(z) k_{l}^{*}(z)\right. \\
& \left.+k_{l-1}(z) k_{l-1}^{*}(z)\right),
\end{aligned}
$$

with

$$
\begin{gathered}
D_{l=0}^{(1) j j}=\frac{1}{2}(\ln (z)-\mathrm{Ci}(2 z)), \\
D_{l=0}^{(2) j j}=\frac{z^{2}}{4}-\frac{z}{4} \sin (2 z)-\frac{1}{8} \cos (2 z), \\
D_{l=1}^{(2) j j}=\frac{z^{2}}{4}+\frac{z}{4} \sin (2 z)+\frac{5}{8} \cos (2 z)+\frac{1}{2} \ln (z)-\frac{1}{2} \operatorname{Ci}(2 z), \\
D_{l=0}^{(1) j h}=\frac{1}{2}(\operatorname{Ei}(1,-2 i z)+\ln (z)), \\
D_{l=0}^{(2) j h}=\frac{z^{2}}{4}+i \frac{z}{4} e^{i 2 z}-\frac{1}{8} e^{i 2 z}, \\
D_{l=1}^{(2) j h}=\frac{z^{2}}{4}-i \frac{z}{4} e^{i 2 z}+\frac{5}{8} e^{i 2 z}+\frac{1}{2} \operatorname{Ei}(1,-2 i z)+\frac{1}{2} \ln (z), \\
(\mathrm{D} 24) \\
D_{l=1}^{(2) h h}=-i \frac{1}{2} z e^{2 i z}+\frac{5}{4} e^{2 i z}+\operatorname{Ei}(1,-2 i z), \\
D_{l=0}^{(1) h h}=\operatorname{Ei}(1,-2 i z),
\end{gathered}
$$

where $\operatorname{Ci}(z)=-\int_{z}^{\infty} d z \cos (z) / z$ is the cosine integral function and $\operatorname{Ei}(1, z)=-\int_{1}^{\infty} d z e^{-z t} / t$ in the first exponential integral function. 
Using these integrals, we have

$$
\begin{aligned}
F_{l m \omega}^{h(3)}(r)= & \int d r^{\prime} g_{l m \omega}\left(r, r^{\prime}\right) s_{l m \omega}^{h(3)}\left[-i 2 z h_{l}^{(1)}(z)\right. \\
& \left.+i \frac{l(l+1)}{z} h_{l}^{(1)}(z)\right]_{z=\omega r^{\prime}} \theta\left(r^{\prime}-r_{C}\right) \\
= & -\frac{i}{\omega^{2}} s_{l m \omega}^{h(3)}\left[\left\{h_{l}^{(1)}(z) D_{l}^{(2) j h}(z)-j_{l}(z) D_{l}^{(2) h h}(z)\right.\right. \\
& \left.-h_{l}^{(1)}(z) D_{l}^{(2) j h}\left(z_{C}\right)\right\} \theta\left(r-r_{C}\right) \\
& \left.-j_{l}(z) D_{l}^{(2) h h}\left(z_{C}\right) \theta\left(z_{C}-z\right)\right],
\end{aligned}
$$

$$
\begin{aligned}
F_{l m \omega}^{j(3)}(r)= & \int d r^{\prime} g_{l m \omega}\left(r, r^{\prime}\right) s_{l m \omega}^{j(3)}\left[-i 2 z j_{l}(z)\right. \\
& \left.+i \frac{l(l+1)}{z} j_{l}(z)\right]_{z=\omega r^{\prime}} \theta\left(r_{C}-r^{\prime}\right) \\
= & -\frac{i}{\omega^{2}} s_{l m \omega}^{j(3)}\left[h_{l}^{(1)}(z) D_{l}^{j j}\left(z_{C}\right) \theta\left(z-z_{C}\right)\right. \\
& +\left\{h_{l}^{(1)}(z) D_{l}^{(2) j j}(z)-j_{l}(z) D_{l}^{(2) j h}(z)\right. \\
& \left.\left.+j_{l}(z) D_{l}^{(2) j h}\left(z_{C}\right)\right\} \theta\left(r-r_{C}\right)\right],
\end{aligned}
$$

where we use $D_{l}^{k k^{*}}=-i 2 D_{l}^{(2) k k^{*}}+i l(l+1) D_{l}^{(1) k k^{*}}$.
[1] C. Cutler and K. S. Thorne, Proceedings of GR16, Durban, South Africa, 2001 (World Scientific, Singapore, 2002).

[2] S. A. Teukolsky, Astrophys. J. 185, 635 (1973); W. H. Press and S. A. Teukolsky, Astrophys. J. 185, 649 (1973); S. A. Teukolsky and W. H. Press, Astrophys. J. 193, 443 (1974).

[3] Y. Mino, Phys. Rev. D 67, 084027 (2003).

[4] Y. Mino, Prog. Theor. Phys. 113, 733 (2005).

[5] Y. Mino, Phys. Rev. D 77, 044008 (2008).

[6] T. Hinderer and E. Flanagan (unpublished).

[7] Y. Mino, Prog. Theor. Phys. 115, 43 (2006).

[8] Recent developments in the issue of the self-force regularization can be found in Part II and Part III of Special issue on Gravitational Radiation from Binary Black Holes: Advances in the Perturbative Approach, edited by Carlos
O. Lousto [Classical Quantum Gravity 22 Number 15 (2005)].

[9] G. Beutler, Methods of Celestial Mechanics (Springer, New York, 2005).

[10] D. Kennefick and A. Ori, Phys. Rev. D 53, 4319 (1996); Y. Mino, M. Sasaki, M. Shibata, H. Tagoshi, and T. Tanaka, Prog. Theor. Phys. Suppl. 128, 1 (1997), Sec. 10.

[11] É. Flanagan (private communication).

[12] J. Matthews and R. L. Walker, Mathematical Methods of Physics, edited by W. A. Benjamin (Menlo Park, 1970), 2nd ed., Sec. 9.5 (Addison-Wesley, Reading, MA, 1970)

[13] F. G. Friedlander, The Wave Equation on a Curved SpaceTime (Cambridge University Press, London, 1976).

[14] C. Beetle, B. Bromely, N. Hernandez, and R. H. Price, Phys. Rev. D 76, 084016 (2007). 